\title{
Quantitative assessment of vegetation layers in tropical evergreen forests of Arunachal Pradesh, Eastern Himalaya, India
}

\author{
Sudhansu Sekhar Dash ${ }^{1, *}$, Samiran Panday ${ }^{2}$, Dinesh Singh Rawat ${ }^{3}$, \\ Vikas Kumar ${ }^{4}$, Subhajit Lahiri ${ }^{3}$, Bipin Kumar Sinha ${ }^{1}$ and Paramjit Singh ${ }^{5}$ \\ ${ }^{1}$ Botanical Survey of India, CGO Complex, Sector-1, Salt Lake, Kolkata 700 064, India \\ ${ }^{2}$ Department of Botany, Budge Budge College, South 24 Parganas 700 137, India \\ ${ }^{3}$ Central National Herbarium, Botanical Survey of India, Howrah 711 103, India \\ ${ }^{4}$ Environmental Technology Division, CSIR-IHBT, Palampur 176 061, India \\ ${ }^{5}$ Department of Botany, Central University of Punjab, Bathinda 151001 , India
}

\begin{abstract}
The present study deals with first-hand information on quantitative assessments of different vegetation layers (viz. trees, saplings, seedlings, shrubs and herbs) collected from 57 permanent plots (size $400 \mathrm{~m}^{2}$ ), established for long-term monitoring of biodiversity and study of functional aspects in Namdapha National Park (NPP), Arunachal Pradesh, Eastern Himalaya, India during 2017. We grouped all the plots into six clusters as study sites. A total of 60 taxa of trees, 67 shrubs and 81 herbs were recorded within 57 plots during the study. The average species richness per site for trees was $20.83 \pm 1.62$, saplings $16.0 \pm 1.15$, seedlings $15.83 \pm 1.35$, shrubs $23.83 \pm 1.58$ and herbs 32.67 \pm 0.92 . Total stem density varied from 117.5 to and $181 \mathrm{ha}^{-1}\left(152.58 \pm 10.04 \mathrm{ha}^{-1}\right)$ for trees (circumference $\geq 31.5 \mathrm{~cm}), 881$ to $3000 \mathrm{ha}^{-1}\left(1652.17 \pm 317.61 \mathrm{ha}^{-1}\right)$ for shrubs and from 76750 to $98545 \mathrm{ha}^{-1}(92032.17 \pm$ $3246.60 \mathrm{ha}^{-1}$ ) for herbs. Tree regeneration status at all the six study sites was 'good' (i.e. density of seedlings $>$ saplings $>$ trees). The distribution of tree stems (circumference $\geq 31.5 \mathrm{~cm}$ ) into different size classes showed highest relative density in the lowest stem size class $(10-20 \mathrm{~cm}$ diameter) which also indicates good tree regeneration in the study area. Dipterocarpus retusus Blume was the most dominant tree species in the core zone area of NNP with 'good' regeneration status.
\end{abstract}

Keywords: Biodiversity, Dipterocarpus retusus, regeneration status, tropical evergreen forests, vegetation layers.

\section{Introduction}

QUANTIFIABLE analysis of community composition is a prerequisite for the precise evaluation of biodiversity, and it plays a central role in conservation biology ${ }^{1-4}$. Quantification of the contemporary composition of Himalayan forests is crucial in order to assess the role of climate

*For correspondence. (e-mail: ssdash2002@gmail.com) change on future species coexistence and to provide baseline data for the long-term monitoring processes and species shift in the Himalayan ranges ${ }^{5,6}$. There is a dearth of studies reporting floristic composition and community structure in tropical evergreen forests of Eastern Himalaya, India. Therefore, a detailed ecological study in these forests is necessary to generate baseline data to assess the different ecological consequences of ongoing and future climate change ${ }^{6}$.

A comprehensive floristic account of the Namdapha National Park (NPP), Arunachal Pradesh was made by Chauhan et al. ${ }^{7}$. This was later supplemented by some notable discoveries, i.e. Sapria himalayana, Begonia tessaricarpa, Ceropegia lucida and Bretschneidera sinensis $^{8-11}$. Nath et al. ${ }^{12}$ analysed the vegetation and tree population structure in a few selected sites of NNP, while Deb and Sundriya ${ }^{13,14}$ observed the tree species gap phase performance, tree regeneration and seedling survival pattern, especially in the buffer zone of the Park. Barbhuiya et $a l .{ }^{15}$ studied the leaf litter decomposition of dominant tree species in NPP. Sarmah et al. ${ }^{16,17}$ documented the ethno-botanical knowledge and natural resource utilization pattern of the tribal living in and around NNP. Besides, plant community structure and tree regeneration from different districts of Arunachal Pradesh were studied by several researchers ${ }^{18-25}$. The aim of this study was to evaluate the species composition, richness, density, basal area and dominance of trees, saplings, seedlings, shrubs and herbs in the western part of NNP.

\section{Materials and methods}

\section{Study area}

The experimental site is situated in the western part of Arunachal Pradesh near the international border of India and Myanmar. The Park occupies an area of $1985 \mathrm{~km}^{2}$ and lies between $27^{\circ} 23^{\prime}-27^{\circ} 39^{\prime} \mathrm{N}$ lat. and $96^{\circ} 15^{\prime}-$ $96^{\circ} 58^{\prime \prime} \mathrm{E}$ long. with altitude ranging from 200 to 
Table 1. General details of the study sites in Namdapha National Park, Eastern Himalaya, India

\begin{tabular}{|c|c|c|c|}
\hline Forest stand or trail & Code given & Coordinates & Altitude ( $\mathrm{m}$ amsl) \\
\hline Haldibari - Hornbill & B1 & $\begin{array}{l}27^{\circ} 31^{\prime} 25.68^{\prime \prime}-27^{\circ} 31^{\prime} 36.89^{\prime \prime} \mathrm{N} \\
96^{\circ} 23^{\prime} 45.37^{\prime \prime}-96^{\circ} 24^{\prime} 59.45^{\prime \prime} \mathrm{E}\end{array}$ & $460-591$ \\
\hline Hornbill - Bulbuliya & $\mathrm{B} 2$ & $\begin{array}{l}27^{\circ} 31^{\prime} 47.35^{\prime \prime}-27^{\circ} 32^{\prime} 20.79^{\prime \prime} \mathrm{N} \\
96^{\circ} 29^{\prime} 13.31^{\prime \prime}-96^{\circ} 27^{\prime} 29.81^{\prime \prime} \mathrm{E}\end{array}$ & $560-745$ \\
\hline 25 mile - 19 mile (west) & $\mathrm{C} 1$ & $\begin{array}{l}27^{\circ} 27^{\prime} 33.19^{\prime \prime}-27^{\circ} 27^{\prime} 56.13^{\prime \prime} \mathrm{N} \\
96^{\circ} 24^{\prime} 35.03^{\prime \prime}-96^{\circ} 25^{\prime} 30.25^{\prime \prime} \mathrm{E}\end{array}$ & $485-612$ \\
\hline 25 mile - Goodbye point (uphill) & $\mathrm{C} 2$ & $\begin{array}{l}27^{\circ} 27^{\prime} 09.64^{\prime \prime}-27^{\circ} 27^{\prime} 43.52^{\prime \prime} \mathrm{N} \\
96^{\circ} 25^{\prime} 39.44^{\prime \prime}-96^{\circ} 25^{\prime} 53.90^{\prime \prime} \mathrm{E}\end{array}$ & $586-951$ \\
\hline 25 mile -27 mile (east) & $\mathrm{C} 3$ & $\begin{array}{l}27^{\circ} 27^{\prime} 49.31^{\prime \prime}-27^{\circ} 27^{\prime} 49.31^{\prime \prime} \mathrm{N}, \\
96^{\circ} 26^{\prime} 02.60^{\prime \prime}-96^{\circ} 26^{\prime} 26.50^{\prime \prime} \mathrm{E}\end{array}$ & $542-589$ \\
\hline 25 mile - riverside (downhill) & $\mathrm{C} 4$ & $\begin{array}{l}27^{\circ} 27^{\prime} 52.62^{\prime \prime}-27^{\circ} 29^{\prime} 23.23^{\prime \prime} \mathrm{N} \\
96^{\circ} 24^{\prime} 17.63^{\prime \prime}-96^{\circ} 27^{\prime} 49.64^{\prime \prime} \mathrm{E}\end{array}$ & $331-487$ \\
\hline
\end{tabular}

$4571 \mathrm{~m}$ amsl. The Park exhibits high diversity of flora ${ }^{7}$ and fauna ${ }^{26}$, and is well known as one of India's pristine biodiversity regions. The vegetation of the Park ranges from lowland tropical forests to alpine scrubs. The lowland tropical rainforest of Namdapha represents the largest remaining Dipterocarpus forests in India ${ }^{13,27}$. NNP exhibits tropical climate experiencing typical monsoon with prolonged rainy season ${ }^{13}$. At lower altitudes, temperature varies from $5^{\circ} \mathrm{C}$ to $35^{\circ} \mathrm{C}$, while it falls to $0^{\circ}$ or below at higher elevations. The annual precipitation ranges from a minimum of $1400 \mathrm{~mm}$ to a maximum of $2500 \mathrm{~mm}, 75 \%$ of which falls between April and October $^{28}$. The average relative humidity remains high $(>60 \%)$ round the year, except during the dry season (NovemberJanuary).

\section{Methodology}

Experimental sites and field work: The present study was conducted in the western part of NNP that serves as a gateway for visitors, forest personnel and residents of Gandhigram and Vijanagar villages (both villages are located on the eastern fringe of the Park). The under-storey vegetation of the study site exhibits dense naturalized bamboo, banana, zingiber, ferns, etc. The high density of the understorey vegetation is one of the major constrains for sampling. Therefore, we adopted stratified random sampling method to study vegetation along six trails (each being 2.5-3 km long) denoted as B1, B2, C1, C2, C3 and $\mathrm{C} 4$. Of these, four sites $(\mathrm{C} 1, \mathrm{C} 2, \mathrm{C} 3$ and $\mathrm{C} 4)$ belong to the core zone, while two (B1 and B2) fall in the buffer zone of the Park. In the core zone, sites $\mathrm{C} 1-\mathrm{C} 4$ begin from the ' 25 mile base camp' area towards four directions, i.e. uphill (C2), downhill (C4), east (C3) and west (C1). B1 represents Haldibari-Hornbill area, while B2 represents Bulbuliya-Hornbill area. Sampling plots (long-term monitoring plots) were established, mapped (GPS) and marked along the track route in each site with a distance of 200-250 m. Table 1 provides details of each site.
Data collection: The field data on different vegetation layers, viz. trees, saplings, seedlings, shrubs and herbs were collected using quadrat method in 2017 . The entire monitoring plot of size $400 \mathrm{~m}^{2}$ was considered as a quadrat for tree vegetation. Within each tree quadrat, four sub-quadrats were nested for saplings (size $25 \mathrm{~m}^{2}$ ), four for seedlings (size $1 \mathrm{~m}^{2}$ ), four for shrubs (size $25 \mathrm{~m}^{2}$ ) and five for herbs (size $1 \mathrm{~m}^{2}$ ). Circumference $(C)$ was used to differentiate tree life stages into mature trees $(C \geq 31.5 \mathrm{~cm}$ at $1.37 \mathrm{~m}$ above ground level), saplings $(C=10.5-$ $31.4 \mathrm{~cm})$ and seedlings $(C<10.5 \mathrm{~cm})$. The number of individuals of each species was counted within the respective quadrats and noted on their respective field-data sheets (separate sheets for trees, saplings, seedlings, shrubs and herbs). Circumference was measured with the help of graduated tape or diameter with callipers. Species occurring within each plot were collected, processed and preserved according to standard protocol ${ }^{29}$.

Data analysis: The collected plant specimens were identified with help of the literature ${ }^{7,30-33}$ and on consultation of different herbaria (ASSAM, ARUN, CAL). The quantifiable data of different vegetation layers of each site were computed for density, basal area and importance value index (IVI) following Misra ${ }^{34}$. In the present study, IVI of herbs was calculated by summing relative frequency and relative density following Rasingam and Parthasarathy ${ }^{35}$. Various diversity indices, viz. dominance index ${ }^{36}$, diversity index ${ }^{37}$, evenness index ${ }^{38}$, Margalef index ${ }^{39}$ and Fisher alpha ${ }^{40}$ were calculated for each site. Individual trees were divided into eight diameter at breast height (DBH) classes, i.e. 10-20, 21-30, 31-40, 41-50, 51$60 \mathrm{~cm}$, and so on. The density-diameter distribution of trees was calculated to understand the pattern of regeneration and structure of each forest stand. The dominancediversity curves ( $d-d$ curves) for six sites were derived from IVI values of different vegetation layers. The regeneration status of tree species was determined on the basis of population size of seedlings and saplings, following Shankar ${ }^{41}$. The statistical analysis was performed using MS Excel and SPSS. 
SPECIAL SECTION:

Table 2. Phytosociological attributes and diversity indices for different vegetation layers at six study sites

\begin{tabular}{|c|c|c|c|c|c|c|c|c|c|c|}
\hline \multirow[b]{2}{*}{ Parameters } & \multicolumn{6}{|c|}{ Study site } & \multicolumn{4}{|c|}{ Statistics $(N=6)$} \\
\hline & B1 & $\mathrm{B} 2$ & $\mathrm{C} 1$ & $\mathrm{C} 2$ & $\mathrm{C} 3$ & $\mathrm{C} 4$ & Maximum & Minimum & Mean & SE \\
\hline \multicolumn{11}{|l|}{ Tree } \\
\hline No. of plots (size $400 \mathrm{~m}^{2}$ ) & 10 & 11 & 10 & 7 & 8 & 11 & 11 & 7 & 9.50 & 0.67 \\
\hline No. of taxa & 21 & 28 & 20 & 16 & 19 & 21 & 28 & 16 & 20.83 & 1.62 \\
\hline No. of individuals & 63 & 80 & 47 & 50 & 47 & 60 & 80 & 47 & 57.83 & 5.22 \\
\hline Total stem density $\left(\mathrm{ha}^{-1}\right)$ & 157 & 181 & 117.5 & 178 & 146 & 136 & 181 & 117.5 & 152.58 & 10.04 \\
\hline Total basal area $\left(\mathrm{m}^{2} \mathrm{ha}^{-1}\right)$ & 34.11 & 34.05 & 22.73 & 72.79 & 45.91 & 18.69 & 72.79 & 18.69 & 38.05 & 7.98 \\
\hline Dominance index & 0.07 & 0.05 & 0.16 & 0.13 & 0.12 & 0.10 & 0.16 & 0.05 & 0.11 & 0.02 \\
\hline Diversity index & 2.80 & 3.10 & 2.43 & 2.38 & 2.55 & 2.66 & 3.10 & 2.38 & 2.65 & 0.11 \\
\hline Evenness index & 0.79 & 0.80 & 0.57 & 0.67 & 0.67 & 0.68 & 0.80 & 0.57 & 0.70 & 0.04 \\
\hline Margalef index & 4.83 & 6.16 & 4.94 & 3.83 & 4.68 & 4.89 & 6.16 & 3.83 & 4.89 & 0.30 \\
\hline Fisher alpha & 11.03 & 15.31 & 13.16 & 8.14 & 11.86 & 11.49 & 15.31 & 8.14 & 11.83 & 0.97 \\
\hline \multicolumn{11}{|l|}{ Tree regeneration status } \\
\hline Fair & 38.71 & 29.03 & 13.64 & 34.62 & 32.14 & 25.93 & 38.71 & 13.64 & 29.01 & 1.03 \\
\hline Good & 16.13 & 25.81 & 22.73 & 15.38 & 14.29 & 29.63 & 29.63 & 14.29 & 20.66 & 2.03 \\
\hline New (only seedling stage) & 9.68 & 3.23 & 13.64 & 19.23 & 14.29 & 14.81 & 19.23 & 3.23 & 12.48 & 3.03 \\
\hline Nil (without regeneration) & 29.03 & 38.71 & 31.82 & 15.38 & 28.57 & 22.22 & 38.71 & 15.38 & 27.62 & 4.03 \\
\hline Poor & 6.45 & 3.23 & 18.18 & 15.38 & 10.71 & 7.41 & 18.18 & 3.23 & 10.23 & 5.03 \\
\hline \multicolumn{11}{|l|}{ Saplings } \\
\hline No. of plots (size $25 \mathrm{~m}^{2}$ ) & 40 & 44 & 40 & 28 & 32 & 44 & 44 & 28 & 38.00 & 2.68 \\
\hline No. of taxa & 18 & 20 & 12 & 14 & 16 & 16 & 20 & 12 & 16.00 & 1.15 \\
\hline No. of individuals & 37 & 51 & 40 & 35 & 32 & 23 & 51 & 23 & 36.33 & 3.77 \\
\hline Total stem density $\left(\mathrm{ha}^{-1}\right)$ & 370 & 463.64 & 400 & 437.5 & 457.14 & 209.09 & 463.64 & 209.09 & 389.56 & 38.91 \\
\hline Total basal area $\left(\mathrm{m}^{2} \mathrm{ha}^{-1}\right)$ & 0.58 & 0.78 & 0.62 & 0.62 & 0.73 & 0.31 & 0.78 & 0.31 & 0.61 & 0.07 \\
\hline Dominance index & 0.08 & 0.08 & 0.21 & 0.12 & 0.1 & 0.08 & 0.21 & 0.08 & 0.11 & 0.02 \\
\hline Diversity index & 2.69 & 2.74 & 1.92 & 2.33 & 2.53 & 2.67 & 2.74 & 1.92 & 2.48 & 0.13 \\
\hline Evenness index & 0.82 & 0.78 & 0.57 & 0.73 & 0.79 & 0.9 & 0.9 & 0.57 & 0.77 & 0.05 \\
\hline Margalef index & 4.71 & 4.83 & 2.98 & 3.66 & 4.33 & 4.78 & 4.83 & 2.98 & 4.22 & 0.30 \\
\hline Fisher alpha & 13.83 & 12.12 & 5.81 & 8.65 & 12.73 & 23.3 & 23.3 & 5.81 & 12.74 & 2.43 \\
\hline \multicolumn{11}{|l|}{ Seedlings } \\
\hline No. of plots (size $1 \mathrm{~m}^{2}$ ) & 40 & 44 & 40 & 28 & 32 & 44 & 44 & 28 & 38.00 & 2.68 \\
\hline No. of taxa & 17 & 20 & 10 & 16 & 17 & 15 & 20 & 10 & 15.83 & 1.35 \\
\hline No. of individuals & 79 & 71 & 64 & 47 & 35 & 50 & 79 & 35 & 57.67 & 6.73 \\
\hline Total stem density $\left(\mathrm{ha}^{-1}\right)$ & 19,750 & 16,136 & 16,000 & 14,687 & 12,500 & 11,363 & 19,750 & 11,363 & $15,072.67$ & $1,216.51$ \\
\hline Total basal area $\left(\mathrm{m}^{2} \mathrm{ha}^{-1}\right)$ & 1.37 & 0.61 & 1.3 & 0.43 & 1.02 & 0.65 & 1.37 & 0.43 & 0.90 & 0.16 \\
\hline Dominance index & 0.09 & 0.19 & 0.26 & 0.14 & 0.1 & 0.11 & 0.26 & 0.09 & 0.15 & 0.03 \\
\hline Diversity index & 2.6 & 2.31 & 1.67 & 2.33 & 2.56 & 2.42 & 2.6 & 1.67 & 2.32 & 0.14 \\
\hline Evenness index & 0.79 & 0.5 & 0.53 & 0.64 & 0.76 & 0.75 & 0.79 & 0.5 & 0.66 & 0.05 \\
\hline Margalef index & 3.66 & 4.46 & 2.16 & 3.9 & 4.5 & 3.58 & 4.5 & 2.16 & 3.71 & 0.35 \\
\hline Fisher alpha & 6.65 & 9.26 & 3.32 & 8.55 & 13.03 & 7.27 & 13.03 & 3.32 & 8.01 & 1.31 \\
\hline \multicolumn{11}{|l|}{ Shrubs } \\
\hline No. of plots (size $25 \mathrm{~m}^{2}$ ) & 40 & 44 & 40 & 28 & 32 & 44 & 44 & 28 & 38.00 & 2.68 \\
\hline No. of taxa & 22 & 22 & 19 & 23 & 28 & 29 & 29 & 19 & 23.83 & 1.58 \\
\hline No. of individuals & 135 & 110 & 300 & 138 & 137 & 97 & 300 & 97 & 152.83 & 30.22 \\
\hline Total stem density $\left(\mathrm{ha}^{-1}\right)$ & 1350 & 1000 & 3000 & 1725 & 1957 & 881 & 3000 & 881 & 1652.17 & 317.61 \\
\hline Total basal area $\left(\mathrm{m}^{2} \mathrm{ha}^{-1}\right)$ & 1.53 & 0.84 & 6.2 & 1.91 & 2.35 & 0.95 & 6.2 & 0.84 & 2.30 & 0.81 \\
\hline Dominance index & 0.14 & 0.08 & 0.29 & 0.23 & 0.16 & 0.14 & 0.29 & 0.08 & 0.17 & 0.03 \\
\hline Diversity index & 2.42 & 2.81 & 1.75 & 2.22 & 2.5 & 2.67 & 2.81 & 1.75 & 2.40 & 0.15 \\
\hline Evenness index & 0.51 & 0.75 & 0.3 & 0.4 & 0.44 & 0.5 & 0.75 & 0.3 & 0.48 & 0.06 \\
\hline Margalef index & 4.28 & 4.47 & 3.16 & 4.47 & 5.49 & 6.12 & 6.12 & 3.16 & 4.67 & 0.42 \\
\hline Fisher alpha & 7.46 & 8.27 & 4.51 & 7.88 & 10.65 & 14.01 & 14.01 & 4.51 & 8.80 & 1.32 \\
\hline \multicolumn{11}{|l|}{ Herbs } \\
\hline No. of plots (size $1 \mathrm{~m}^{2}$ ) & 50 & 55 & 50 & 35 & 40 & 55 & 55 & 35 & 47.50 & 3.35 \\
\hline No. of taxa & 35 & 31 & 32 & 31 & 31 & 36 & 36 & 31 & 32.67 & 0.92 \\
\hline No. of individuals & 489 & 542 & 459 & 307 & 331 & 443 & 542 & 307 & 428.50 & 37.39 \\
\hline Total stem density $\left(\mathrm{ha}^{-1}\right)$ & 97,800 & 98,545 & 91,800 & 76,750 & 94,571 & 92,727 & 98,545 & 76,750 & $92,032.17$ & $3,246.60$ \\
\hline Dominance index & 0.13 & 0.09 & 0.06 & 0.07 & 0.06 & 0.06 & 0.13 & 0.06 & 0.08 & 0.01 \\
\hline Diversity index & 2.69 & 2.87 & 3.03 & 2.93 & 3.03 & 3.17 & 3.17 & 2.69 & 2.95 & 0.07 \\
\hline Evenness index & 0.42 & 0.57 & 0.65 & 0.6 & 0.67 & 0.66 & 0.67 & 0.42 & 0.60 & 0.04 \\
\hline Margalef index & 5.49 & 4.77 & 5.06 & 5.24 & 5.17 & 5.74 & 5.74 & 4.77 & 5.25 & 0.14 \\
\hline Fisher alpha & 8.63 & 7.14 & 7.83 & 8.61 & 8.37 & 9.26 & 9.26 & 7.14 & 8.31 & 0.30 \\
\hline
\end{tabular}




\section{Results and discussion}

The variation in phytosociological attributes of different Himalayan forests is driven by environmental variables, including soil condition, slope angles, species composition, elevation, regional climate and topography. In the present study, we found slight to noticeable variations in the phytosociological attributes and diversity indices of all the five vegetation layers, viz. trees, saplings, seedlings, shrubs and herbs from one site to another. The hierarchical cluster analysis (using the Bray-Curtis similarity, single linkage) is depicted in Figure 1 using tree species composition in 57 plots nested across the six sites. Two sites in the buffer zone area, viz. B1 and B2 showed maximum similarity in tree species composition, while maximum dissimilarity was observed between two sites in the core zone area, viz. $\mathrm{C} 1$ and $\mathrm{C} 2$. A total of 60

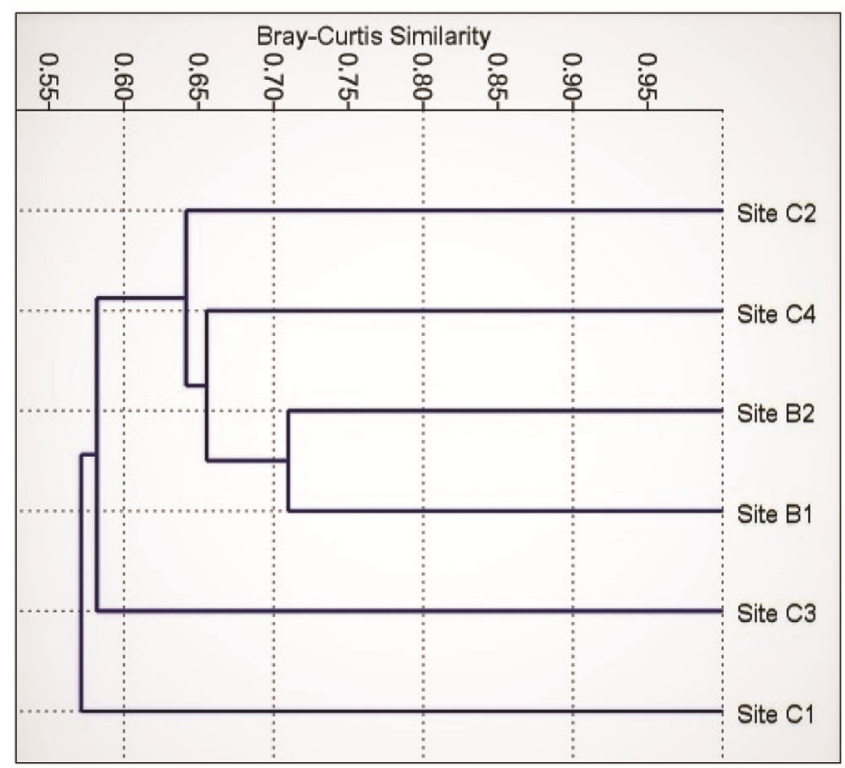

Figure 1. Hierarchical cluster analysis (Bray-Curtis, single linkage) of six study sites using tree species composition.

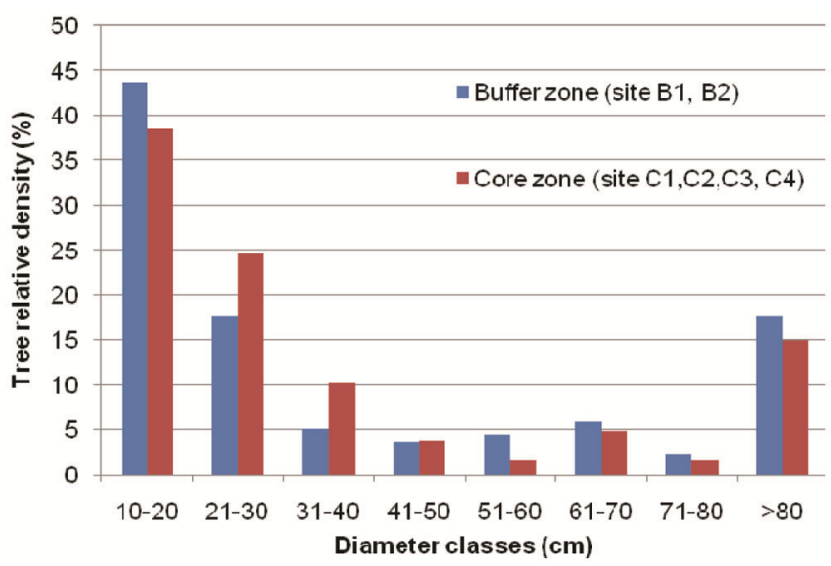

Figure 2. Distribution of trees into different size classes. species of trees, 67 shrubs and 81 herbs were recorded from the 57 plots. The species richness (SR) among the sites varied from 16 to 28 for trees, 19 to 29 for shrubs, 12 to 20 for saplings, 10 to 20 for seedlings and 31 to 36 for herbs (Table 2), which was much higher than the SR reported by Das et al. ${ }^{24}$ for shrubs, saplings, seedlings and herbs (except trees layers that were found to be similar) from Pinus merkusii-dominated forests of Anjaw, Arunachal Pradesh. Behera and Kushwaha ${ }^{18}$ observed high SR for trees $(\mathrm{cbh} \geq 15 \mathrm{~cm}$ ) from Subansiri district, Arunachal Pradesh than that in the present study (trees, $\mathrm{cbh} \geq 31.5 \mathrm{~cm})$.
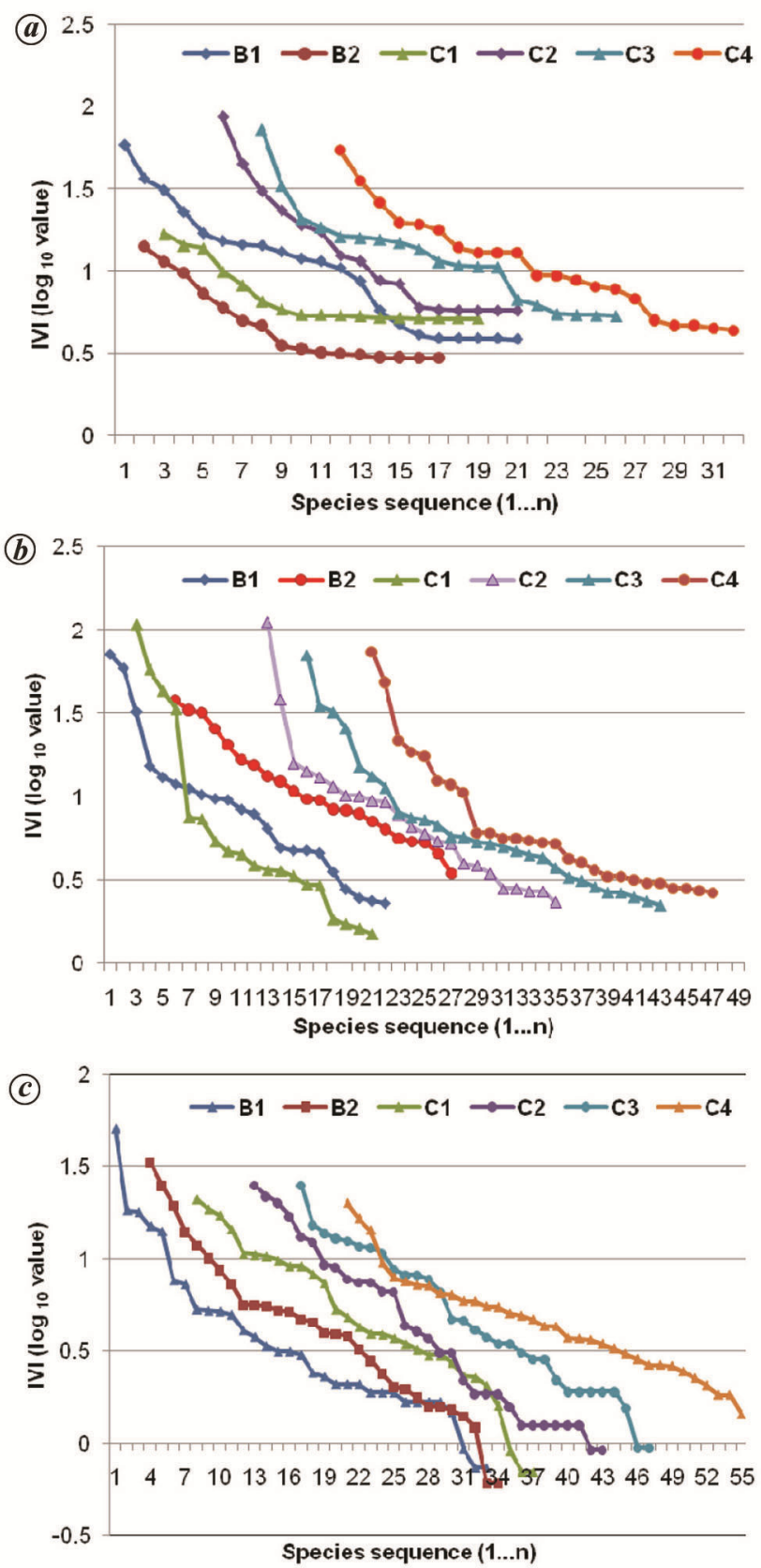

Figure 3. Dominance-diversity curves for different sites (a) tress, (b) shrubs and $(c)$ herbs. 
Table 3. Four dominant taxa (with IVI value) at different study sites in tree, shrub and herb layers

\begin{tabular}{|c|c|c|c|}
\hline Site & Tree layer & Shrub layer & Herb layer \\
\hline \multirow[t]{4}{*}{ B1 } & Dysoxylum excelsum Blume (58.52) & Bambusa tulda Roxb. (71.53) & $\begin{array}{l}\text { Amischotolype mollissima (Blume) } \\
\text { Hassk. }(50.46)\end{array}$ \\
\hline & Mesua ferrea L. (36.58) & $\begin{array}{l}\text { Miliusa roxburghiana Hook.f. \& } \\
\text { Thomson (59.22) }\end{array}$ & Chloranthus elatior Link (18.19) \\
\hline & Cleidion javanicum Blume (31.16) & $\begin{array}{l}\text { Strobilanthes secunda T. Anderson } \\
\quad(32.37)\end{array}$ & Begonia palmata D.Don (17.79) \\
\hline & Dipterocarpus retusus Blume (22.88) & Sabia lanceolata Colebr. (15.39) & $\begin{array}{l}\text { Myrioneuron nutans Wall. ex } \\
\text { Hook. f. (15.01) }\end{array}$ \\
\hline \multirow[t]{4}{*}{$\mathrm{B} 2$} & Altingia excelsa Noronha (26.27) & $\begin{array}{l}\text { Boehmeria macrophylla Hornem. } \\
\quad \text { (37.92) }\end{array}$ & $\begin{array}{l}\text { Elatostema sessile J.R.Forst. \& G. } \\
\text { Forst. (24.89) }\end{array}$ \\
\hline & Dysoxylum excelsum Blume (25.21) & $\begin{array}{l}\text { Saprosma ternatum (Wall.) Hook.f. } \\
\quad(33.18)\end{array}$ & Selaginella monospora Spring (19.39) \\
\hline & $\begin{array}{l}\text { Colona floribunda (Wall. ex Kurz) Craib } \\
\text { (24.02) }\end{array}$ & $\begin{array}{l}\text { Miliusa roxburghiana Hook.f. \& } \\
\text { Thomson (31.97) }\end{array}$ & $\begin{array}{l}\text { Myrioneuron nutans Wall. ex Hook. f. } \\
\quad(13.93\end{array}$ \\
\hline & Dipterocarpus retusus Blume (21.45) & $\begin{array}{l}\text { Myxopyrum smilacifolium (Wall.) } \\
\text { Blume }(25.60)\end{array}$ & $\begin{array}{l}\text { Baliospermum calycinum Müll. Arg. } \\
\text { (11.73) }\end{array}$ \\
\hline \multirow[t]{4}{*}{$\mathrm{C} 1$} & Dipterocarpus retusus Blume (143.33) & Bambusa tulda Roxb. (108.09) & Carex baccans Nees (20.99) \\
\hline & $\begin{array}{l}\text { Mallotus roxburghianus Müll. Arg. } \\
(23.14)\end{array}$ & $\begin{array}{l}\text { Musa velutina } \mathrm{H} . \text { Wendl. \& Drude } \\
\quad(58.25)\end{array}$ & $\begin{array}{l}\text { Amischotolype mollissima (Blume) } \\
\text { Hassk. (18.59) }\end{array}$ \\
\hline & $\begin{array}{l}\text { Knema cinerea var. glauca (Blume) Y.H. } \\
\quad \text { Li (16.81) }\end{array}$ & Calamus erectus Roxb. (43.29) & $\begin{array}{l}\text { Pollia secundiflora (Blume) Bakh.f. } \\
\quad(17.23)\end{array}$ \\
\hline & Ficus altissima Blume(14.35) & $\begin{array}{l}\text { Ensete glaucum (Roxb.) Cheesman } \\
\quad(33.66)\end{array}$ & Centotheca lappacea (L.) Desv. (14.48) \\
\hline \multirow[t]{4}{*}{$\mathrm{C} 2$} & Dipterocarpus retusus Blume (87.44) & Bambusa tulda Roxb. (111.95) & Elatostema platyphyllum Wedd. (24.95) \\
\hline & Shorea assamica Dyer (45.13) & Calamus erectus Roxb. (38.43) & Selaginella monospora Spring (21.69) \\
\hline & Quercus lamellosa $\mathrm{Sm}$. (30.74) & Smilax perfoliata Lour. (15.94) & $\begin{array}{l}\text { Amischotolype mollissima (Blume) } \\
\text { Hassk. (20.06) }\end{array}$ \\
\hline & $\begin{array}{l}\text { Terminalia myriocarpa Van Heurck \& } \\
\text { Müll. Arg. (23.53) }\end{array}$ & $\begin{array}{l}\text { Strobilanthes secunda T. Anderson } \\
\quad(14.22)\end{array}$ & $\begin{array}{l}\text { Begonia hatacoa Buch.-Ham. ex D. } \\
\text { Don (16.91) }\end{array}$ \\
\hline \multirow[t]{4}{*}{$\mathrm{C} 3$} & Dipterocarpus retusus Blume (73.02) & Bambusa tulda Roxb. (70.99) & Phrynium pubinerve Blume (24.91) \\
\hline & $\begin{array}{l}\text { Terminalia myriocarpa Van Heurck \& } \\
\text { Müll. Arg. (33.28) }\end{array}$ & Musa balbisiana Colla (35.12) & Elatostema platyphyllum Wedd. (15.29) \\
\hline & $\begin{array}{l}\text { Duabanga grandiflora (DC.) Walp. } \\
\quad(20.71)\end{array}$ & $\begin{array}{l}\text { Musa velutina } \mathrm{H} \text {. Wendl. \& Drude } \\
\quad(32.27)\end{array}$ & $\begin{array}{l}\text { Hedychium coccineum Buch.-Ham. ex } \\
\text { Sm. (13.70) }\end{array}$ \\
\hline & Cleidion javanicum Blume (18.56) & Calamus erectus Roxb. (25.78) & $\begin{array}{l}\text { Rhynchotechum ellipticum (Wall. ex } \\
\text { D. Dietr.) A. DC. (12.79) }\end{array}$ \\
\hline \multirow[t]{4}{*}{$\mathrm{C} 4$} & Dipterocarpus retusus Blume (54.21) & Bambusa tulda Roxb. (74.30) & $\begin{array}{l}\text { Amischotolype mollissima (Blume) } \\
\text { Hassk. (20.01) }\end{array}$ \\
\hline & $\begin{array}{l}\text { Magnolia hodgsonii (Hook. f. \& Thomson) } \\
\text { H. Keng (35.46) }\end{array}$ & Calamus erectus Roxb. (48.83) & $\begin{array}{l}\text { Piper hymenophyllum (Miq.) Wight } \\
\quad(16.56)\end{array}$ \\
\hline & Kydia calycina Roxb. (26.21) & Saurauia napaulensis DC. (21.98) & $\begin{array}{l}\text { Begonia hatacoa Buch.-Ham. ex } \\
\text { D. Don (14.29) }\end{array}$ \\
\hline & Bombax ceiba L. (19.78) & Leea indica (Burm. f.) Merr. (18.58) & Psychotria denticulata Wall.(9.54) \\
\hline
\end{tabular}

The average density per site recorded in the present study was $152.58 \pm 10.04 \mathrm{ha}^{-1}$ for trees, $1652.17 \pm$ $317.61 \mathrm{ha}^{-1}$ for shrubs and $92032.2 \pm 3246.6 \mathrm{ha}^{-1}$ for herbs (Table 2) which was in agreement with the density, range of trees and shrubs reported from other forests of Arunachal Pradesh by Rana and Gairola ${ }^{21}$ and Das et $a l .^{24}$, but herb density in the present study was much higher comparatively. Pearson's correlation analysis revealed that the tree density was negatively related with density of shrubs and herbs, while positively $(P>0.05)$ with the density of saplings and seedlings (Appendix 1). The tree density organized into different size classes represented reverse $J$-shaped distribution (Figure 2) because a higher stem density occurred in the lower size classes $(10-20,21-30 \mathrm{~cm})$ and it decreased in higher classes. Such distribution in natural forest stands indicates a stable population with good regeneration status ${ }^{42-44}$. Occurrence of $17.52 \%$ individuals in the core zone area and $14.97 \%$ in the buffer zone area in the highest diameter class $(>80 \mathrm{~cm})$ in the present study is indicative of old and climax forest that has maintained good regeneration status and reproductive success over the ages.

In the present study, Dipterocarpus retusus was identified as the dominant species in tree layers at all the four sites in the core zone area of NNP (Table 3), while the two sites in the buffer zone, viz. B1 and B2 were dominated by Dysoxylum excelsum and Altingia excelsa respectively. The shrub layer was dominated by Bambusa 
Table 4. Comparison of diversity $\left(H^{\prime}\right)$, dominance $(D)$ and evenness $(E)$ indices in the present study with those of other studies from Arunachal Pradesh

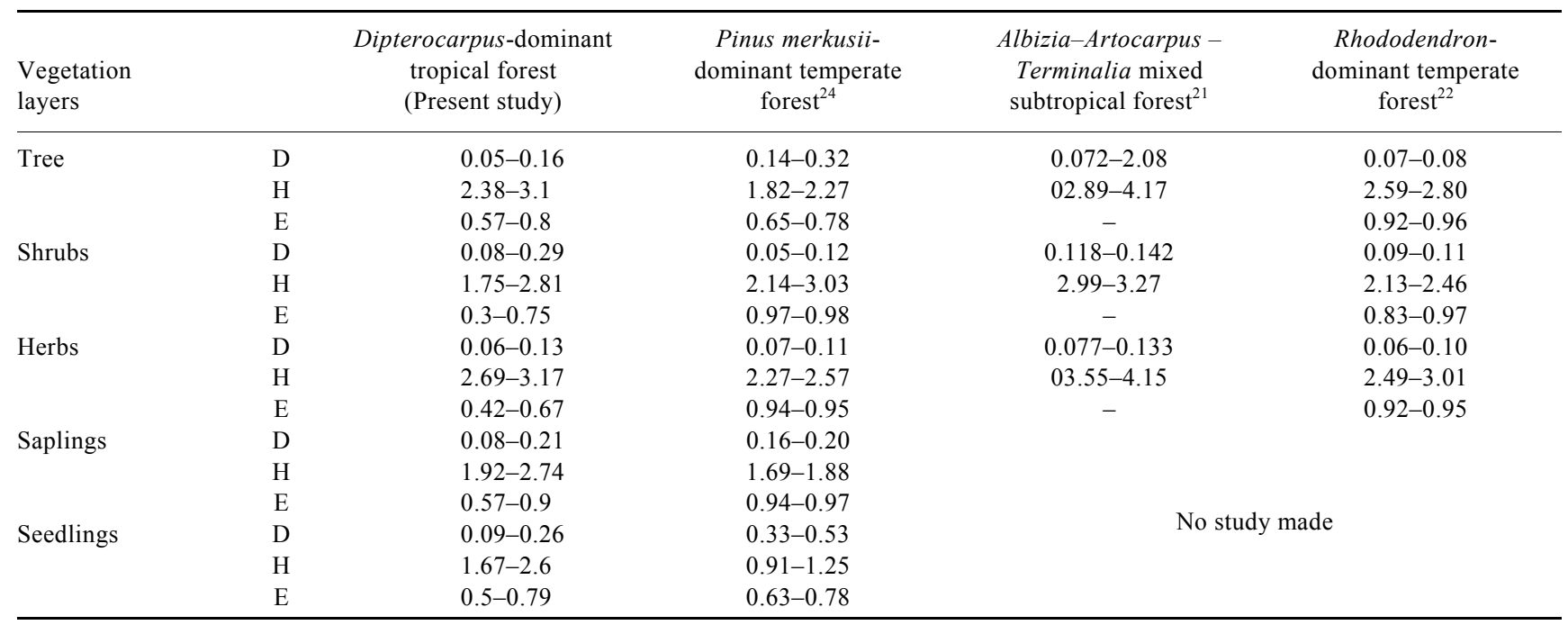

Appendix 1. Pearson's correlation between the phytosociological parameters of different vegetation layers

\begin{tabular}{|c|c|c|c|c|c|c|c|c|c|c|c|c|c|c|}
\hline & Tden & Tbas & Tdom & Tdiv & Saden & Sabas & Sadiv & Seden & Sebas & Sediv & Shden & Shbas & Shdiv & Hden \\
\hline Tbas & 0.65 & 1.00 & & & & & & & & & & & & \\
\hline Tdom & -0.61 & 0.15 & 1 & & & & & & & & & & & \\
\hline Tdiv & 0.45 & -0.36 & -0.94 & 1 & & & & & & & & & & \\
\hline Saden & 0.46 & 0.57 & 0.02 & 0.05 & 1 & & & & & & & & & \\
\hline Sabas & 0.44 & 0.40 & -0.13 & 0.24 & 0.97 & 1 & & & & & & & & \\
\hline Sadiv & 0.54 & -0.06 & -0.90 & 0.74 & -0.19 & -0.08 & 1 & & & & & & & \\
\hline Sebas & -0.60 & -0.45 & 0.16 & -0.07 & 0.02 & 0.08 & -0.28 & 0.53 & 1 & & & & & \\
\hline Sediv & 0.50 & 0.28 & -0.61 & 0.34 & -0.09 & -0.06 & 0.86 & -0.06 & -0.19 & 1 & & & & \\
\hline Shden & -0.57 & 0.04 & 0.83 & -0.67 & 0.36 & 0.28 & -0.93 & 0.10 & 0.51 & -0.73 & 1 & & & \\
\hline Shbas & -0.69 & -0.19 & 0.79 & -0.58 & 0.19 & 0.14 & -0.94 & 0.12 & 0.55 & -0.85 & 0.96 & 1 & & \\
\hline Shdiv & 0.54 & -0.07 & -0.84 & 0.74 & -0.15 & -0.03 & 0.95 & -0.23 & -0.46 & 0.75 & -0.92 & -0.92 & 1 & \\
\hline Hden & -0.20 & -0.73 & -0.57 & 0.73 & -0.08 & 0.13 & 0.45 & 0.26 & 0.52 & 0.17 & -0.23 & -0.12 & 0.41 & 1 \\
\hline Hdiv & -0.54 & -0.26 & 0.53 & -0.42 & -0.45 & -0.46 & -0.28 & -0.91 & -0.32 & -0.30 & 0.14 & 0.21 & -0.06 & -0.21 \\
\hline
\end{tabular}

Tden, Tree stem density; Tbas, Tree basal area; Tdom, Tree dominance index; Tdiv, Tree diversity index; Saden, Sapling density; Sabas, Sapling basal area; Sadiv, Sapling diversity index; Seden, Seedling density; Sebas, Seedling basal area; Sediv, Diversity index; Shden, Shrub density; Shbas, Shrub basal area; Shdiv, Shrub diversity index; Hden, Herb density, Hdiv, Herb diversity index.

tuld $a$ at all the sites in the core zone and in site B2 in the buffer zone, while shrub layer in site B1 was dominated by Boehmeria macrophylla. In the core zone Carex baccans, Elatostema platyphyllum, Elatostema platyphyllum and Amischotolype mollissima were recorded as the dominant herbs at sites $\mathrm{C} 1, \mathrm{C} 2, \mathrm{C} 3$ and $\mathrm{C} 4$ respectively, while in the buffer zone one site was dominated by Elatostema sessile and the other (B1) by Amischotolype mollissima.

The $d-d$ curves clealy delimit the vegetational layers along different gradients and also show their dominance due to various ecological factors. Figure 3 shows the $d-d$ curves for trees, shrubs and herbs respectively versus log normal values at different sites. The different diversity indices recorded from NNP were compared with those reported from other Eastern Himalayan forests (Table $4)^{21,22,24}$. The sites in the buffer zone area represented slightly higher values of SR, diversity, evenness, Margalef index and Fisher alpha index in comparison to the core zone area in the present study, while inverse results were obtained for dominance index. The tree dominance index showed significant negative correlation with the diversity index $(r=0.94, P<0.05)$. Similar relation between dominance and diversity index was reported in some other Himalayan forests ${ }^{45-48}$.

The existing natural population of trees at all the six forest stands exhibited 'good' regeneration status (i.e. density of seedlings $>$ saplings $>$ trees) in general during the study period, which varied at species level (i.e. same taxon showed different status at different sites). In 
SPECIAL SECTION:

Table 5. Regeneration status of tree species in the Namdapha National Park, Arunachal Pradesh during 2017

\begin{tabular}{|c|c|c|c|c|c|c|}
\hline \multirow[b]{2}{*}{ Tree taxon } & \multicolumn{6}{|c|}{ Study site } \\
\hline & $\mathrm{B} 1$ & B2 & $\mathrm{C} 1$ & $\mathrm{C} 2$ & $\mathrm{C} 3$ & $\mathrm{C} 4$ \\
\hline Actinodaphne obovata (Nees) Blume & Fair & Nil & - & - & - & - \\
\hline Aesculus assamica Griff. & New & Nil & - & Nil & - & Fair \\
\hline Ailanthus excelsa Roxb. & Good & Nil & New & New & - & Poor \\
\hline Alangium chinense (Lour.) Harms & Fair & - & Nil & - & - & - \\
\hline Albizia procera (Roxb.) Benth. & - & Nil & - & New & - & - \\
\hline Alnus nepalensis D.Don & - & - & - & - & Nil & - \\
\hline Altingia excelsa Noronha & Nil & Fair & - & - & - & - \\
\hline Aralia armata (Wall. ex G.Don) Seem. & Fair & Good & - & - & Fair & - \\
\hline Balakata baccata (Roxb.) Esser & - & - & Poor & Poor & - & - \\
\hline Bischofia javanica Blume & Fair & Nil & - & - & - & Nil \\
\hline Bombax ceiba L. & - & - & - & - & - & Nil \\
\hline Bridelia glauca Blume & - & Fair & - & New & Fair & Fair \\
\hline Callicarpa arborea Roxb. & - & - & New & - & Good & - \\
\hline Caryota urens $\mathrm{L}$. & - & - & - & Nil & Fair & - \\
\hline Castanopsis indica (Roxb. ex Lindl.) A.DC. & New & Good & - & - & - & - \\
\hline Cinnamomum bejolghota (Buch.-Ham.) Sweet & Fair & Good & New & Poor & Fair & New \\
\hline Cinnamomum glanduliferum (Wall.) Meisn. & Nil & - & - & - & Nil & New \\
\hline Cleidion javanicum Blume & Fair & Good & - & Fair & Fair & Poor \\
\hline Colona floribunda (Kurz) Craib & - & - & - & Fair & - & - \\
\hline Crateva religiosa G.Forst. & - & Nil & - & - & - & - \\
\hline Dipterocarpus retusus Blume & Good & Nil & Good & Good & Fair & Good \\
\hline Duabanga grandiflora (DC.) Walp. & - & - & - & - & Fair & - \\
\hline Dysoxylum excelsum Blume & Good & Good & Fair & Good & Good & Good \\
\hline Elaeocarpus rugosus Roxb. ex G.Don & - & Fair & - & Fair & - & - \\
\hline Engelhardtia spicata Lechen ex Blume & - & - & Nil & - & Nil & Nil \\
\hline Erythrina arborescens Roxb. & - & - & - & - & - & Nil \\
\hline Evodia fraxinifolia (Hook.) Benth. & - & - & - & - & Nil & - \\
\hline Ficus altissima Blume & Fair & Nil & Good & New & & Good \\
\hline Ficus auriculata Lour. & - & - & - & - & New & - \\
\hline Ficus nervosa B.Heyne ex Roth & - & - & Nil & - & - & - \\
\hline Glochidion khasicum (Müll.Arg.) Hook.f. & Nil & Nil & - & - & - & Fair \\
\hline Grewia eriocarpa Juss. & - & - & New & Fair & Nil & - \\
\hline Gynocardia odorata R.Br. & & Nil & - & - & - & - \\
\hline Knema cinerea var. glauca (Blume) Y.H. Li & - & Fair & Nil & Good & Poor & Good \\
\hline Kydia calycina Roxb. & Nil & - & - & - & New & Fair \\
\hline Lasianthus lucidus Blume & Nil & Fair & - & Fair & - & - \\
\hline Litsea monopetala (Roxb.) Pers. & - & Fair & Nil & - & - & - \\
\hline Macaranga denticulata (Blume) Müll.Arg. & Fair & - & Good & Fair & New & New \\
\hline Machilus gamblei King ex Hook. f. & & - & Poor & Fair & Poor & Good \\
\hline Magnolia hodgsonii (Hook.f. \& Thomson) H.Keng & Good & Good & - & Fair & Nil & Good \\
\hline Mallotus roxburghianus Müll.Arg. & - & - & Good & - & New & Fair \\
\hline Mangifera sylvatica Roxb. & Nil & Fair & - & - & - & - \\
\hline Melia azedarach L. & - & - & - & - & - & Nil \\
\hline Mesua ferrea $\mathrm{L}$. & Good & - & Poor & Poor & - & New \\
\hline Ocotea lancifolia (Schott) Mez & Poor & Good & - & - & Nil & Fair \\
\hline Olea dioica Roxb. & - & - & - & - & - & - \\
\hline Oreocnide integrifolia (Gaudich.) Miq. & - & New & Poor & - & Good & - \\
\hline Photinia integrifolia Lindl. & - & - & - & - & Poor & - \\
\hline Puzunglo (localy identified) & - & Fair & - & - & - & - \\
\hline Quercus lamellosa $\mathrm{Sm}$. & Nil & - & - & Nil & - & - \\
\hline Quercus semiserrata Roxb. & Fair & Nil & Fair & Good & - & Nil \\
\hline Saprosma ternatum (Wall.) Hook.f. & Poor & - & - & - & - & - \\
\hline Saurauia armata Kurz & Fair & Good & Fair & New & Good & Good \\
\hline Schima wallichii Choisy & New & - & Nil & - & - & - \\
\hline Shorea assamica Dyer & Fair & Fair & - & Poor & - & Fair \\
\hline Styrax serrulatus Roxb. & - & - & - & Nil & Nil & - \\
\hline Terminalia myriocarpa Van Heurck \& Müll. Arg. & Fair & Nil & Good & Fair & Fair & Good \\
\hline Toona ciliate M.Roem. & Nil & - & - & - & - & - \\
\hline Turpinia pomifera (Roxb.) DC. & Nil & Poor & Nil & - & - & - \\
\hline Uvaria dioeca Roxb. & - & - & - & - & Fair & - \\
\hline
\end{tabular}


species-level regeneration, majority of the species (29.01\%) showed 'fair' regeneration status while $20.66 \%$ exhibited 'good' regeneration status and $10.23 \pm 5.03 \%$ species showed 'poor' regeneration status. About $12.48 \%$ of the species were only represented by seedlings ('new' regeneration) and $27.62 \%$ showed 'Nil' regeneration (Table 5). Inter- and intra-species competition, dense and virgin canopy cover (large and medium-sized trees) and abundant undergrowth of herbaceous layer could be reasons affecting the regeneration, particularly for species with 'poor' and 'Nil' regenerating status. However, the present contribution is part of ongoing work in the park. Further progress with increasing the number of monitoring plots may highlight the possible reasons for regeneration failure by some species.

\section{Conclusion}

The present study has provided data on tree, shrub and herbaceous communities in selected forest stands of NNP. Similar type of data from different parts of the Park can be generated which will be helpful in assessing the effect of climate change and other ecological impacts. The phytosociological attributes and ecological indices show that NNP has sustained a good floral diversity, with (good) overall regeneration status. The reasons for 'poor' and 'Nil' regenerating species need to be evaluated for their proper conservation.

1. Oosting, H. J., The Study of Plant Communities, W.H. Freeman and Company, San Francisco, CA, USA, 1956.

2. Huang, W., Pohjonen, V., Johansson, S., Nashanda, M., Katigula, M. I. L. and Luukkanen, O., Species diversity, forest structure and species composition in Tanzanian tropical forests. For. Ecol. Manage., 2003, 173(1-3), 11-24.

3. Eilu, G. and Obua, J., Ecology tree condition and natural regeneration in disturbed sites of Bwindi Impenetrable Forest National Park, south western Uganda. Trop. Ecol., 2005, 46(1), 99-111.

4. Mwavu, E. N. and Witkowski, Ed. T. F., Population structure and regeneration of multiple-use tree species in a semi-deciduous African tropical rainforest: Implications for primate conservation. For. Ecol. Manage., 2009, 258(5), 840-849.

5. Sharma, C. M., Mishra, A. K., Krishan, R., Tiwari, O. P. and Rana, Y. S., Impact of climate on structure and composition of ridge top forests in Garhwal Himalaya. Taiwania, 2016, 61(2), 61-69.

6. Rawat, D. S., Dash, S. S., Sinha, B. K., Kumar, V., Banerjee, A. and Singh, P., Community structure and regeneration status of tree species in eastern Himalaya: A case study from Neora Valley National Park, West Bengal, India. Taiwania, 2018, 63(1), 16-24.

7. Chauhan, A. S., Singh, P. K. and Singh, D. K., Contribution to the Flora of Namdapha, Arunachal Pradesh, Botanical Survey of India, Calcutta, 1996.

8. Adhikari, D., Arunachalam, A., Majumdar, M., Sarmah, R. and Khan, M. L., A report on the rare root parasite (Sapria himalayana Griffith.) in Namdapha National Park in northeast India. Curr. Sci., 2003, 85, 1668-1669.

9. Kumar, A. and Amadudin, M., Rediscovery of an endemic and endangered plant (Begonia tessaricarpa C.B. Clarke) from Arunachal Pradesh, India, after a century. Curr. Sci., 2006, 91(8), 997998.
10. Khandal, D., More, M., Kataria, G. and Kambale, S., Ceropegia lucida-rediscovery and new distribution record for Arunachal Pradesh, India. Curr. Sci., 2017, 113(11), 2077.

11. Kumar, V., Dash, S. S., Panday, S., Lahiri, S., Sinha, B. K. and Singh, P., Akaniaceae: A new family record for flora of India and lectotypification of the name Bretschneidera sinensis. Nelumbo, 2017, 59(1), 1-9.

12. Nath, P. C., Arunachalam, A., Khan, M. L., Arunachalam, K. and Barbhuiya, A. R., Vegetation analysis and tree population structure of tropical wet evergreen forests in and around Namdapha national park, north-east India. Biodivers. Conserv., 2005, 14, 2109-2136.

13. Deb, P. and Sundriyal, R. C., Tree species gap phase performance in the buffer zone area of Namdapha National Park, eastern Himalaya, India. Trop. Ecol., 2007, 48(2), 209-225.

14. Deb, P. and Sundriyal, R. C., Tree regeneration and seedling survival patterns in old-growth lowland tropical rainforest in Namdapha National Park, north-east India. For. Ecol. Manage., 2008, 255, 3995-4006.

15. Barbhuiya, A. R., Arunachalam, A., Nath, P. C., Khan, M. L. and Arunachalam, K., Leaf litter decomposition of dominant tree species of Namdapha. J. For. Res., 2008, 13, 25-34.

16. Sarmah, R., Adhikari, D., Majumder, M. and Arunachalam, A., Indigenous technical knowledge of Lisus with reference to natural resource utilization in the far-eastern villages of Arunachal Pradesh, India. Indian J. Tradit. Knowl., 2006, 5(1), 51-56.

17. Sarmah, R., Arunachalam, A., Majumder, M., Melkania, U. and Adhikari, D., Ethno-medicobotany of Chakmas in Arunachal Pradesh, India. Indian For., 2006, 132(4), 474-484.

18. Behera, M. D., Kushwaha, S. P. S., Roy, P. S., Srivastava, S., Singh, T. P. and Dubey, R. C., Comparing structure and composition of coniferous forests in Subansiri district, Arunachal Pradesh. Curr. Sci., 2002, 82, 70-76.

19. Duchok, R., Kent, K., Devi, K. A., Paul, A. and Khan, M. L., Population structure and regeneration status of medicinal tree Illicium griffithii in relation to disturbance gradients in temperate broadleaved forest of Arunachal Pradesh. Curr. Sci., 2005, 89(4), 673676.

20. Behera, M. D. and Kushwaha, S. P. S., An analysis of altitudinal behavior of tree species in Subansiri district, eastern Himalaya. Biodivers. Conserv., 2007, 16, 1851-1865.

21. Rana, C. S. and Gairola, S., Forest community structure and composition along an elevational gradient of Parshuram Kund area in Lohit district of Arunachal Pradesh, India. Nat. Sci., 2009, 8(1), $44-52$.

22. Bharali, S., Paul, A., Khan, M. L. and Singha, L. B., Species diversity and community structure of a temperate mixed rhododendron forest along an altitudinal gradient in west Siang district of Arunachal Pradesh, India. Nat. Sci., 2011, 9, 125-140.

23. Yam, G. and Tripathi, O. P., Tree diversity and community characteristics in Talle Wildlife Sanctuary, Arunachal Pradesh, Eastern Himalaya, India. J. Asia Pac. Biodivers., 2016, 9, 160165.

24. Das, A. K., Singha, L. B. and Khan, M. L., Community structure and species diversity of Pinus merkusii Jungh. \& de Vriese forest along an altitudinal gradient in eastern Himalaya, Arunachal Pradesh, India. Trop. Ecol., 2017, 58(2), 397-408.

25. Paul, A., Khan, M. L. and Das, A. K., Population structure and regeneration status of rhododendrons in temperate mixed broadleaved forests of western Arunachal Pradesh, India. Geol. Ecol. Landsc., 2018, 3(3), 168-186.

26. Ghosh, A. K., Qualitative Analysis of Faunal Resources of Proposed Namdapha Biosphere Reserve, Zoological Survey of India, Calcutta, 1987.

27. Proctor, J., Haridasan, K. and Smith, G. W., How far does lowland evergreen tropical rainforest go? Global Ecol. Biogeogr., 1998, 7, $141-146$. 
28. Arunachalam, A., Sarmah, R., Adhikari, D., Majumder, M. and Khan, M. L., Anthropogenic threats and biodiversity conservation in Namdapha nature reserve in the Indian eastern Himalayas. Curr. Sci., 2004, 87, 447-454.

29. Jain, S. K. and Rao, R. R., A Handbook of Field and Herbarium Methods, Today \& Tomorrow's Printers \& Publishers, New Delhi, 1997.

30. Chowdhery, H. J., Giri, G. S., Pal, G. D., Pramanik, A. and Das, S. K., Materials for the flora of Arunachal Pradesh. Ranunculaceae Dipsacaceae. In Flora of India, Series 2, Botanical Survey of India, Kolkata, 1996, vol. 1, p. 692.

31. Chowdhery, H. J., Giri, G. S., Pal, G. D., Pramanik, A. and Das, S. K. Materials for the flora of Arunachal Pradesh. AsteraceaeCeratophyllaceae. In Flora of India, Series 2, Botanical Survey of India, Kolkata, 2008, vol. 2, p. 492.

32. Chowdhery, H. J., Giri, G. S., Pal, G. D., Pramanik, A. and Das, S. K., Materials for the flora of Arunachal Pradesh. Hydrocharitaceae - Poaceae. In Flora of India, Series 2, Botanical Survey of India, Kolkata, 2009, vol. 3, p. 491.

33. Dash, S. S. and Singh, P., Flora of Kurung Kumey District, Aruanchal Pradesh, Botanical Survey of India, Kolkata, 2017, p. 778.

34. Misra, R., Ecology Workbook, Oxford and IBH, Calcutta, 1998, p. 242.

35. Rasingam, L. and Parthasarathy, N., Diversity of understory plants in undisturbed and disturbed tropical lowland forests of Little Andaman Island, India. Biodivers. Conserv., 2009, 18, 1045-1065.

36. Simpson, E. H., Measurement of diversity. Nature, 1949, 163, 688 .

37. Shannon, C. E. and Wiener, W. E., The Mathematical Theory of Communities, University of Illinois Press, Urbana, USA, 1963, p. 117.

38. Buzas, M. A. and Gibson, T. G., Species diversity: benthonic forminifera in western North Atlantic. Science, 1969, 163, 7-75.

39. Margalef, R., Information theory in ecology. General Systems, 1958, 3, 36-71. (Trans1. from Mem. R. Acad. Cienc. Artes Barc., 1958, 32, 373-449).

40. Fisher, R. A., Corbet, A. S. and Williams, C. B., The relation between the number of species and the number of individuals in a random sample of an animal population. J. Anim. Ecol., 1943, 12, $42-58$.
41. Shankar, U., A case of high tree diversity in a Sal (Shorea robusta) dominated lowland forest of eastern Himalaya: Floristic composition, regeneration and conservation. Curr. Sci., 2001, 81, 776-786.

42. Saxena, A. K. and Singh, J. S., Tree population structure of certain Himalayan forest associations and implications concerning their future composition. Vegetatio, 1984, 58(2), 61-69.

43. Khan, M. L., Rai, J. P. N. and Tripathi, R. S., Population structure of some tree species in disturbed and protected subtropical forests of northeast India. Acta Oecol., 1987, 8(3), 247-255.

44. Rawat, D. S., Tiwari, P., Das, S. K. and Tiwari, J. K., Tree species composition and diversity in montane forests of Garhwal Himalaya in relation to environmental and soil properties. J. Mt. Sci., 2020, 17(12), 3097-3111.

45. Bhuyan, P., Khan, M. L. and Tripathi, R. S., Tree diversity and population structure in undisturbed and human-impacted stands of tropical wet evergreen forest in Arunachal Pradesh, eastern Himalayas India. Biodivers. Conserv., 2003, 12, 1753-1773.

46. Semwal, S., Nautiyal, B. P. and Bhatt, A. B., Dominance diversity patterns and regeneration status of moist temperate forests in Garhwal, part of north-west Himalaya, India. Taiwan J. For. Sci., 2008, 23(4), 351-364.

47. Gairola, S., Sharma, C. M., Suyal, S. and Ghildiyal, S. K., Composition and diversity of five major forest types in moist temperate climate of the western Himalayas. For. Stud. China, 2011, 13(2), 139-153.

48. Rawat, D. S., Tiwari, J. K., Tiwari, P., Nautiyal, M., Parveen, M. and Singh, N., Tree species richness, dominance and regeneration status in western Ramganga valley, Uttarakhand Himalaya, India. Indian For., 2018, 144(7), 595-603.

ACKNOWLEDGEMENTS. We thank the PCCF, Forest Department, Arunachal Pradesh for granting permission to conduct this study, and the Project Director, Namdapha Tiger Reserve and National Park, Miao for logistic support throughout the study. We also thank the Ministry of Environment, Forest and Climate Change, Government of India for financial support through a project (NMHS/2015-16/LG-05).

doi: $10.18520 / \mathrm{cs} / \mathrm{v} 120 / \mathrm{i} 5 / 850-858$ 